\title{
Effect of partial discharges on the operating condition of high- voltage insulators
}

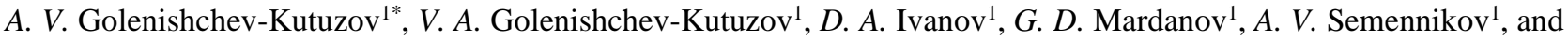 \\ L. V. Ahmetvaleeva ${ }^{1}$ \\ ${ }^{1}$ Kazan State Power Engineering University, Kazan, Russia
}

\begin{abstract}
The features of the dielectric rod defects and the rod-electrod defects of polymer insulators were investigated by using the developed complex method of contactless remote diagnostics of high-voltage insulators health, which includes the initial detection of local areas with elevated electric field gradients as well as measurement of a set of partial discharges characteristics. The radiation of extra-large partial discharges was detected and partial discharges features were investigated. According to the measurement results, a diagram was constructed for the electro-physical processes that accompany the emission of extralarge partial discharges; and the reasons for their occurrence were established.
\end{abstract}

\section{Introduction}

A variety of organic polymers have been used as electrical insulating materials for electric power apparatus and cables. A large amount of work has been done on degradation of the insulating materials caused by partial discharges (PD) occurring at various defects in the polymer insulator itself and at the interface between electrodes and the insulating materials [1-6]. PD degradation is one of the most significant factors deciding the life of the insulation system. Much remains unknown about the mechanisms of the PD degradation. In order to clarify the mechanism of PD degradation, a deeper understanding of the fundamental properties of $\mathrm{PD}$ is essential.

As is known from theoretical and experimental studies $[7,8]$, surface cracks on or bulk cavities in the dielectric rod are the main types of defects in highvoltage insulators (HVI). Another type of defects is loose connection between the core and the end terminal. Defects at the junction points of the rod with the end terminal are most dangerous, since the electric field in the air gap of microscopic size can significantly exceed the average field strength across the sample; emission from the cathode (electrode) will also significantly increase the current through the defect, thereby reducing the threshold of electrical breakdown. Since a strong field gradient emerges near the rod surface in the rodendterminal contact containing a microscopic air layer, prolonged service accelerates aging and decomposition of the dielectric medium that deteriorate the electrical strength of the insulator. Therefore, it is important to separate these basic types of defects and their locations in the insulator [9].

Such characteristics of PDs as a drop in the emergence threshold and an increase in the intensity and repetition rate can serve as indicators of the emergence of primary defects in the HVI. The main problems encountered when using the method of recording PDs in HVIs are the lack of developed apparatus and the absence of PD parameters normed for actual HVIs. In recent years, a number of methods for remotely measuring the characteristics of PDs in HVIs have been proposed that make it possible to establish the degree of insulator defectiveness but fail to determine the type and location of the defect and only provide partial-discharge characteristics averaged over the entire sample. As follows from explored sources of information, no methods of contactless remote monitoring based on the recording and analysis of partial discharges have so far been proposed that allow determining the type and location of defects in in-service high-voltage insulators.

\section{Measurement of characteristics and analysis of partial discharges parameters}

The Department of Industrial Electronics and Lighting Engineering (IELE) at Kazan State Power Engineering University (KSPEU) developed a method and a device that allow one to remotely measure PD characteristics and, based on them, determine the degree of HVI operability (Figure 1). In the developed method, narrowbeam electromagnetic and acoustic antennas are used to receive signals from partial discharges in the form of electromagnetic pulses that are further amplified with broadband amplifiers and displayed on oscilloscope screen. The partial-discharge pulses are then fed to an analog-to-digital converter and further to a digital information-processing device. Partial-discharge signals synchronized with the high-voltage phase are

\footnotetext{
* Corresponding author: campoce6e@gmail.com
} 
accumulated and distributed over narrow phase intervals in the PC memory. Then this phase distribution for the number of pulses and intensity is compared with the prerecorded distribution of counterpart signals from a reference insulator. A dedicated piece of software detects signals exceeding a level that is safe for normal operation, and these signals are used to identify insulators with defects and decide on the possibility of their further functioning.

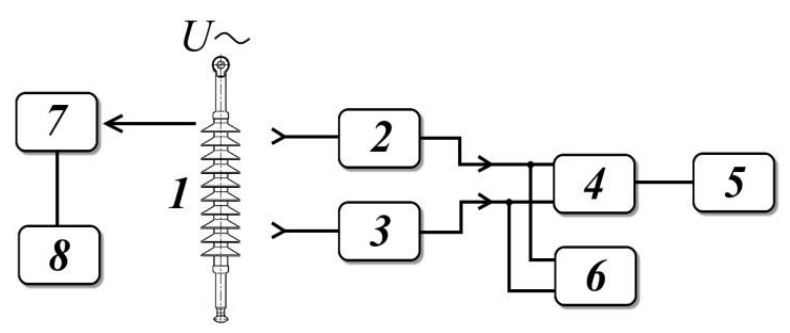

Fig. 1. Flow chart of the measurement device: (1) high-voltage insulator; (2) receiver of electromagnetic signals; (3) receiver of acoustic signals; (4) analog-to-digital converter; (5) personal computer; (6) two-channel oscilloscope; (7) electrooptical sensor; (8) electric field recorder.

Local overpotentials in high-voltage insulators, transformer coils, and cables can indicate defects that lead in the long run to damage or even the destruction of insulating elements. It is therefore vital to monitor local overpotentials during the service life of insulation by remote and noncontact means. However, such means of control have yet to find wide application in high-voltage systems, though there have been attempts to create them [10].

In [11], we proposed localizing areas with increased intensity of the electrical field and measuring the gradients of field intensity with an electrooptical sensor in order to monitor the intensity of the electrical field via the reflectivity of a laser beam from this sensor as a value proportional to the intensity of the electrical field for the noncontact remote diagnostics of the state of high-voltage insulators. The electrooptical sensor is in this case preliminarily calibrated by placing it into a measured alternating electrical field. The average alternating electrical field intensities corresponding to the high working voltage and the limit values of electrical field intensity gradients that do not lead to electrical breakdown or insulator flashover are subsequently determined during testbench measurements for each type of high-voltage insulator subject to diagnostics. The electrooptical sensor, mounted on a dielectric rod and connected with a laser emitter and a photoreceiver through a polarization discriminator and a fiber light guide, is then used to scan the surface of the high-voltage insulator. At the same time, special computer software is used to detect the location of the electrooptical sensor on the insulator's surface and the electrical field intensity corresponding to this location, and to measure the normal and tangential components of the gradients of electrical field intensity. The spatial distribution of the increased gradients of electrical field intensity, both normal and tangential to the surface, are finally compared to the intensity distribution recorded earlier for a reference high-voltage insulator.

Basic scheme of the device for noncontact remote diagnostics of the state of high-voltage insulators is shown in the Figure 2. Electrooptical sensor 2 and fiber light guide 4 contain no metallic elements, and electrooptical sensor 2 is manufactured so as to allow its positioning directly in an alternating electrical field.

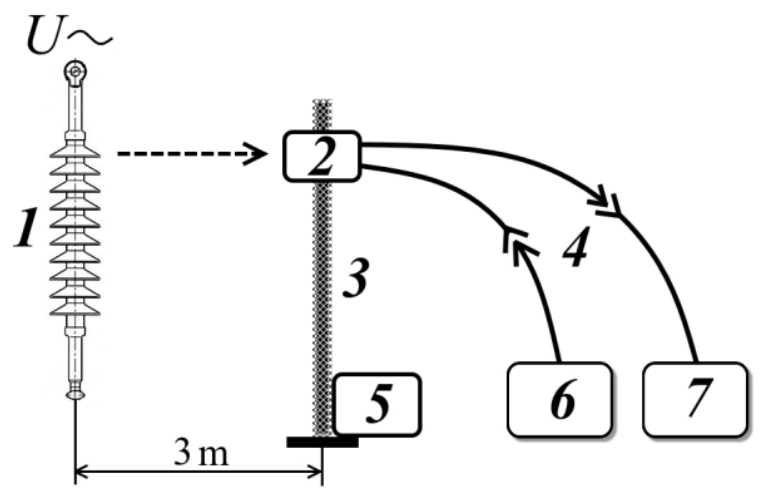

Fig. 2. Basic scheme of the device: (1) high-voltage insulator; (2) electrooptical sensor; (3) scanning block; (4) fiber light guide; (5) moving module; (6) consecutively connected laser emitter; (7) photoreceiver.

\section{Results of inspecting the condition of high-voltage insulators at substations}

This combined method has been trialled on a batch of LK 70/35 polymer insulators, IOS 110/400 porcelain highvoltage insulators (PHVI), and model samples. Particular attention was paid to samples that had defects of two types, damages to the rod sheath and damages to the rod-end-terminal contact. These types of defects were first assessed visually, and then a set of PD characteristics was measured using the IELE KSPEU test bench (Figure 3) [12].

Our analysis of the specific features observed in the set of PD characteristics made it possible to establish significant differences in the phase distributions of the intensity and the number of PD pulses for the investigated HVIs. Some of the PD characteristics had typical distributions that correspond to the previously considered defects in the form of cracks in model samples made of electrical porcelain or defects at the surface of PHVI and polymer HVI rods [13].

Our survey made it possible to divide PHVIs into three groups according to the degree of defectiveness, and, consequently, operability. The first group included fully functional PHVIs with the minimum intensity $(q<200 \mathrm{pC})$ and number $(N<500)$ of PDs located in phase intervals near 90 and $270^{\circ}$; the second group was conditionally operable PHVIs not requiring replacement and containing PDs with $q \leq 300 \mathrm{pC}$ and $N \leq 1000$ and $\Delta \varphi$ in the intervals of $50-80^{\circ}$ and $220-250^{\circ}$ of operating phase voltage; and the third consisted of PHVIs retaining efficiency only for a certain limited period of time and requiring special attention or even replacement during next routine inspections. The latter PHVIs differ sharply from the first two groups by a higher intensity of 

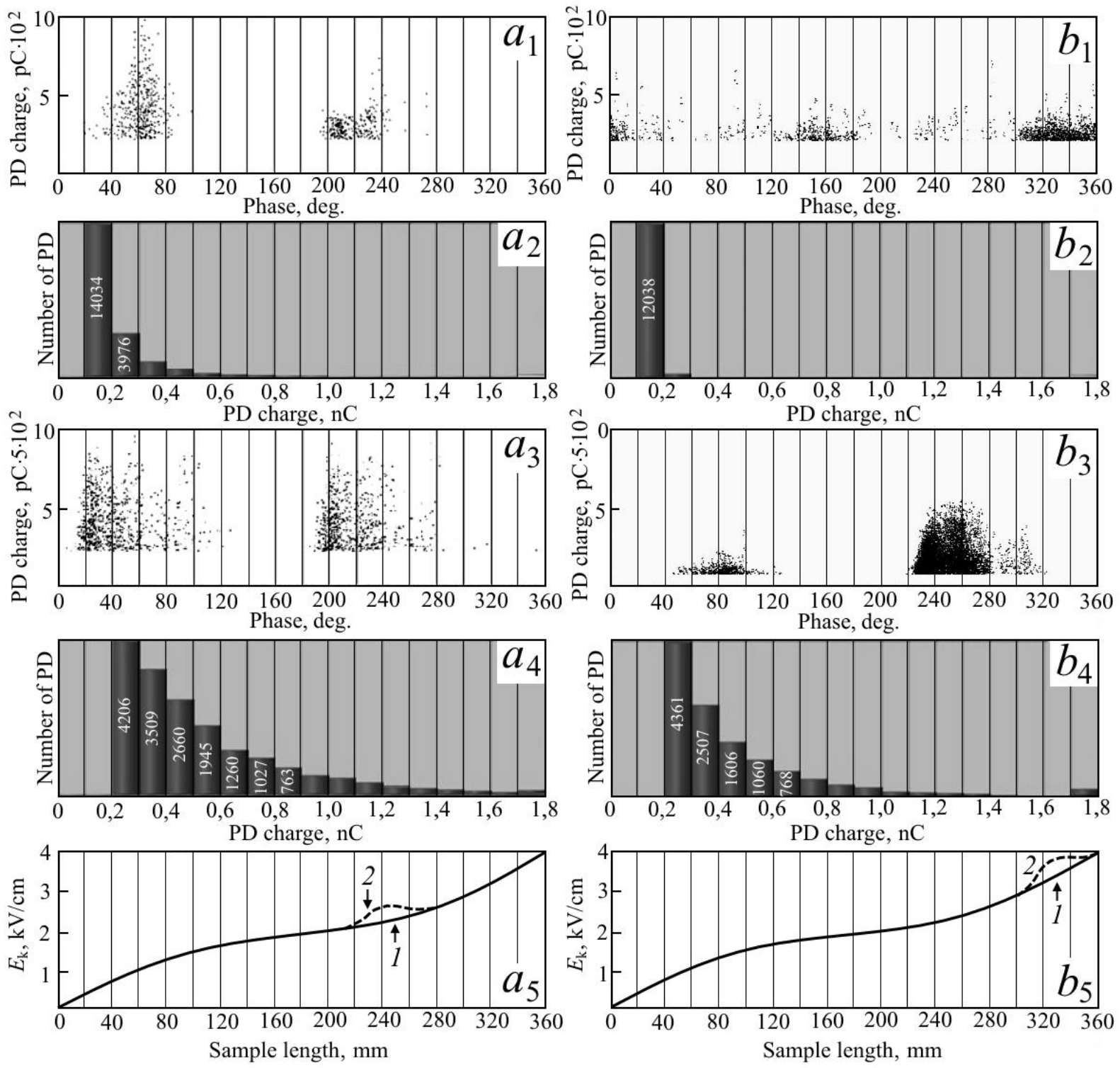

Fig. 3. PD characteristics $\left(a_{1}-a_{4}, b_{1}-b_{4}\right)$ and distribution of the electric fields on the defects $\left(a_{5}, b_{5}\right)$ for polymeric LK $70 / 35$ insulators: $a$-defect at rod, $b$-rod-terminal contact defect.

powerful PDs (up to $2 \mathrm{nC}$ ), an increased (2-5 times) number of PDs, and a shift $\Delta \varphi$ of PD intervals relative to the high-voltage phase periods.

Since PDs mainly occur due to ionization in the air gap, their parameters are determined by the size of the crack. Uniform distribution of the number and intensity of PDs between two network half-periods indicates a symmetric location of the gap surface in the applied field [1], which fully matches PD characteristics for the first two PHVI groups. The small intensity of PDs perfectly fits the small size of cracks, which insignificantly affect the electrophysical state of PHVIs.

As already examined theoretically [14,15] and experimentally [16] for model samples, electrical breakdown in an insulator defect of another type (the third group) is more complicated than in the case of the surface breakdown of a crack. PDs on a defect between the rod and the end terminal are characterized by the broadening and shift of the phase intervals of emission, an increase in the number of PDs in negative voltage half-periods, and a change in the shape of PD pulses. In this case, the breakdown in a contact consisting of the electrode, the gap, and the rod occurs in the air gap due to a field component normal to the gap, by emitting electrons from the electrode (cathode). As a result of electronic bombardment of the dielectric-rod surface, an induced charged field of ions with a density of up to $10^{-}$ ${ }^{6}-10^{-5} \mathrm{C} / \mathrm{cm}$ is formed at this surface, which has sufficient time to change sign when the applied voltage phase changes by $180^{\circ}$.

Thus, the onset and intensity of a PD are determined by the sign and strengths of the applied and induced fields when their sum exceeds the electrical breakdown field. As calculations and measurements have shown, it is after the applied field enters the negative phase that the condition for the summation of the two fields is fulfilled, accompanied by the generation of the strongest PDs, while destructive interference between the applied 
and induced fields leads to a net field decrease and to an extension of the phase band of PD emission. This effect was theoretically considered almost at the same time as our experiments reported in [14].

The intensity of PD also depends on the area and width of the gap between the electrode and the dielectric rod. Since under the conditions of long-term service, these parameters are of a random and uncontrolled nature, it is the intensity and number of PDs that are asymmetric with respect to phase halfperiods that can be determined by the parameters of the defect. Moreover, under prolonged operation of such a defective contact (due to electronic-thermal processes [17]), accelerated aging and even damage may occur in the dielectric, which ultimately reduces the electrical strength of the insulator.

It should be noted that the induced field $E_{i}$ due to a PD also affects PD characteristics, shifting the ending of PD emission intervals toward larger phase angles, but since PD generation processes under a sign change in the applied voltage are symmetric (because $E_{i}<E_{\mathrm{a}}$ ) for small defect sizes, the intensity and number of PDs change little. The difference in the shape of positive and negative PD pulses is due to the fact that the former are created by positive charges induced in the air-gap plasma while the latter are mainly initiated by the faster flow of induced electrons.

\section{Conclusions}

The results of the survey of the working condition of HVIs conducted using the developed two-channel method in the test-bench and field versions have proved the possibility of remotely testing the degree of operability of HVIs under in-service conditions and detecting the type and location of the most hazardous defects. This became possible after supplementing the earlier established diagnostic criteria of remote testing, distinguishing serviceable HVIs from defective ones, with additional features that allow one to determine the type and location of defects. These include a significant broadening and change of PD emission phase intervals, an increase in the number and intensity of PDs during negative high-voltage half-periods in comparison with positive ones, and a decrease in the width of negative PD pulses.

This work was supported by the Russian Foundation for Basic Research, project no. 18-08-00203.

\section{References}

[1] V.Ya. Ushakov, Isolyatsiya ustanovok vysokogo napryazheniya (Insulation in High-Voltage Facilities) (Moscow: Energoatomizdat) 496 (1994)

[2] R. Bartnicas, IEEE Transactions on Dielect. and Elect. Insulation 9, 763-808 (2002)

[3] V.P. Vdoviko, Chastichnye razryady $v$ diagnostirovanii vysokovol'tnogo oborudovaniya (Partial Discharge in the Diagnosis of High-Voltage Equipment) (Novosibirsk: Science) 156 (2007)
[4] A.S. Gaivoronsky, Chief Power Engineer 2, 23-27 (2010)

[5] R. Salustiano, R.M. Capelini, S.R. de Abreu, M.L.B. Martinez, I.C. Tavares, G.M.F. Ferraz, M.A.A. Romano Int. Conf. on High Voltage Engineering and Application (ICHVE) (Poznan: IEEE) 1-4 (2014)

[6] A.V. Golenishchev-Kutuzov, V.A. Golenishchev-Kutuzov, G.D. Mardanov, R.A. Khusnutdinov, and I.A. Evdokimov, Russian J. of Nondestructive Testing 52 8, 478-483 (2016)

[7] A.V. Golenishchev-Kutuzov, GolenishchevV.A. Kutuzov, A.I. Evdokimov, A.Yu. Chernomashentsev, A Method of Montactless Remote Diagnostics of the State of High-Voltage Insulators (RF Patent No 2483315) (2011)

[8] C. Pan, Y. Meng, K. Wu, Z. Han, K. Qin, Y. Cheng, J. of Phys. D Appl. Phys. 44, 255201-208 (2011) [9] H.A. Illias, G. Chen, P.L. Lewin J. of Phys. D Appl. Phys. 44, 245202-216 (2011)

[10] A.V. Golenishchev-Kutuzov, V.A. Golenishchev-Kutuzov, R.A. Khusnutdinov, G.D. Mardanov Electrotekhnika 3, $72-73$ (2017)

[11] A.V. Golenishchev-Kutuzov, V.A. Golenishchev-Kutuzov, G.D. Mardanov, A.V. Semennikov Bulletin of the Russian Academy of Sciences: Physics 83, 125-127 (2019)

[12] A.V. Golenishchev-Kutuzov, V.A. Golenishchev-Kutuzov, D.A. Ivanov, G.D. Mardanov and A.V. Semennikov The Method of Non-Contact Remote Diagnostics of High-Voltage Insulators (RF Patent for invention No 2679759) (2018)

[13] A.V. Golenishchev-Kutuzov, V.A. Golenishchev-Kutuzov, D.A. Ivanov, G.D. Mardanov and A.V. Semennikov Russian J. of Nondestructive Testing 54 10, 10-14 (2018)

[14] G. Callender, I.O. Golosnoy, P.Rapisarda and P.L. Lewin J. of Phys. D Appl. Phys. 51, 125601-624 (2018)

[15] A.L. Kupershtokh and D.I.Karpov J. of Phys.: Conf. Ser. 754, 102006-11 (2016)

[16] K. Wu, C. Pan, Y. Meng, Y. Cheng IEEE Transactions on Dielect. and Elect. Insulation 20, pp 612-619 (2013)

[17] Yu.N. Vershinin Electronno-teplovye $i$ distantsionnye protsessy pri elektricheskom proboe tverdykh dielektrikov (Electronic-Thermal and Remote Processes in the Electrical Breakdown of Solid Dielectrics) (Yekaterinburg: Ural Branch, Russ. Acad. Sci.) 260 (2000) 